\title{
SUFFICIENT CONDITIONS THAT A MAPPING BE OPEN
}

\author{
CHARLES J. TITUS
}

Let $M$ and $N$ be connected oriented $n$-manifolds. A mapping $f: M \rightarrow N$ is said to be quasi-open (Whyburn [4, p. 110]) provided that for any image point $\pi$ and any open set $U$ containing a compact component of $f^{-1}(\pi), \pi$ is interior to $f(U)$ relative to $N$. Openness in this paper is always relative to either $M$ or $N$.

The importance of the concept of quasi-open mappings is seen partly in the facts that every open mapping ( $U$ open implies $f(U)$ open) is quasi-open; every light quasi-open mapping is open; the quasi-open mappings are closed under almost uniform convergence. Also it is often considerably easier to find convenient sufficient conditions for quasi-open mappings than for open mappings. For details on these remarks see Whyburn [4, pp. 110-113] and [3]. Finally, quasi-open mappings have the weak maximum modulus property and, when $M$ and $N$ are planes, are characterized by the minimum modulus property Whyburn [5, p. 48].

However, there exist mappings which are quasi-open but which are not the almost uniform limit of $(n-2)$-light open mappings (preimage of every point has dimension not greater than $n-2$ ). For such an example define the mapping of the $x y$-plane into the $u v$-plane by $u=x, v=x y$. The mapping is sense-preserving on the half-plane $x>0$ and sense-reversing when $x<0$ and it is quasi-open. But it is certainly not the almost uniform limit of light open mappings for the light open mappings are either always sense-preserving or always sensereversing.

Let $W$ denote the set of points of $M$ at which $f$ is locally 1-1 and sense preserving and $Z$ the set of points of $M$ at which $f$ is not locally 1-1. A mapping $f: M \rightarrow N$ will belong to the class $F$ provided that

(i) $W \cup Z=M$, (thus $f$ never reverses sense),

(ii) $f(Z)$ contains no open sets,

(iii) $f$ is constant on every component of the interior of $Z$.

In this paper it will be shown that any nontrivial mapping in $F$ is quasi-open (and by hypothesis nowhere sense reversing) and that certain convenient differential inequalities imply that a mapping is in $F$. The proof of Theorem 1 makes use of the following result of Whyburn [4, Theorem 4.2, p. 111]:

Theorem (Whyburn). If $A$ and $B_{0}$ are locally connected generalized

Received by the editors December 22, 1958. 
continua, a mapping $f(A)=B \subset B_{0}$ is quasi-open if and only if for each conditionally compact region $R$ in $A, f(R) \cap Q$ not empty implies $f(R) \supset Q$ where $Q$ is any component of $B_{0}-f(\operatorname{Fr}(R))$. (Here $\bar{R}$ is the closure of $R$ and $\operatorname{Fr}(R)=\bar{R}$-interior of $R$ ).

Some properties of the degree of a mapping will also be used. The degree, $\operatorname{deg}(f, \pi, U)$ is defined relative to any conditionally compact open set $U \subset M$ and a point $\pi \in N-f(F r(U))$. It has the following properties (see e.g., [1, pp. 474-497]): $\pi \notin f(U)$ implies $\operatorname{deg}(f, \pi, U)$ $=0 ; \operatorname{deg}(f, \pi, U) \quad \neq 0$ implies $\pi \in f(U) ; U_{1} \cap U_{2}$ empty implies $\operatorname{deg}\left(f, \pi, U_{1}\right)+\operatorname{deg}\left(f, \pi, U_{2}\right)=\operatorname{deg}\left(f, \pi, U_{1} \cup U_{2}\right) ; \operatorname{deg}(f, \pi, U)$ is constant on each component of $N-f(F r(U)) ; f$ a $1-1$ sense preserving mapping on $U$ implies $\operatorname{deg}(f, \pi, U)=1$ for all $\pi \in f(U)$.

Lemma 1. Let $f$ be a mapping in $F, R$ a conditionally compact region in $M$ with $\operatorname{Fr}(R)$ not empty. Then, for any point $\pi \in N-f(F r(R))$, $\operatorname{deg}(f, \pi, R)>0$ if and only if $\pi \in f(R)$.

Proof. All that has to be shown is that $\pi \in f(R) \operatorname{implies} \operatorname{deg}(f, \pi, R)$ $>0$ since the proof in the other direction is a property of the degree for arbitrary mappings.

Let $B$ be a component of $N-f(F r(R))$ and $\pi \in f(R) \cap B$. It will first be shown that $f(R) \cap B$ contains a nonempty open set. Now $C=f^{-1}(B)$ $\cap R$ is nonempty and open. If $f(C)=f(R) \cap B$ contains no open set, $C$ is in $Z$. One then sees easily that the boundary of each component of $C$ maps into $f(\operatorname{Fr}(R))$. Thus, by (iii), $f(C) \subset f(\operatorname{Fr}(R))$, a contradiction, and $f(R) \cap B$ must contain an open set. Since, by (ii), $f(Z)$ contains no open sets there exist points $\pi^{\prime} \in f(R) \cap B$ such that $\pi^{\prime} \notin f(Z)$. Thus, $f^{-1}\left(\pi^{\prime}\right)$ is an isolated set in $W \cap R$. Since $\bar{W} \cap \bar{R}$ is compact and since no limit point of $f^{-1}\left(\pi^{\prime}\right)$ can be in $Z, f^{-1}\left(\pi^{\prime}\right)$ is finite. The degree, $\operatorname{deg}\left(f, \pi^{\prime}, C\right)$ is therefore computable and, using (i), is seen to be positive. Thus, by the choice of $\pi^{\prime}, \operatorname{deg}(f, \pi, R)=\operatorname{deg}\left(f, \pi^{\prime}, R\right)$ and since it follows easily that $\operatorname{deg}\left(f, \pi^{\prime}, R\right)=\operatorname{deg}\left(f, \pi^{\prime}, C\right)$ the proof of the lemma is complete.

Theorem 1. Let $f \in F$, then either $f$ is quasi-open or $M$ is compact and $f(M)$ is a point.

Proof. Let $R$ be a conditionally compact region in $M$ with $\operatorname{Fr}(R)$ not empty. Let $Q$ be any component of $N-f(F r(R))$. If $f(R) \cap Q$ is not empty then by the lemma, $\pi \in f(R) \cap Q \operatorname{implies} \operatorname{deg}(f, \pi, R)$ is positive. By the constancy of the degree on each component $Q$ one has then that $f(R) \cap Q$ nonempty implies $Q \subset f(R)$.

If $M$ has the property that every conditionally compact region 
$R \subset M$ has $\operatorname{Fr}(R)$ nonempty the Whyburn Theorem implies that $f$ is quasi-open.

If, on the other hand, there is a conditionally compact region $R \subset M$ such that $\operatorname{Fr}(R)$ is empty then $M$ is compact. Select $R=M-p$, $p$ a point, and one has by the previous arguments that $(N-f(p))$ $\cap f(R)$ is empty or $N-f(p) \subset f(R)$. In the first case $f(M)$ is a point. In the second case $f$ is quasi-open and the proof of Theorem 1 is complete.

Corollary. If $M$ is compact and $f$ is in $F$ then either $f(M)$ is a point or $f(M)=N$ and the degree of $f$ is a positive constant.

Note that for any quasi-open mappings with $M$ compact, $f(M)=N$. However, there exist such mappings where the degree of $f$ is zero. (For example, the mapping $f, f\left(S^{2}\right)=S^{2}$, given by collapsing the equator to a point and then the upper and lower parts onto $S^{2}$ as sense preserving and sense reversing homeomorphisms respectively.) This corollary then points up a special property of the mappings in $F$ over the quasi-open mappings. It seems likely, partly because of this, that the mappings in $F$ are the almost uniform limits of light open mappings (which also have the positive degree property). The author has not been able to show this.

An easy consequence, Corollary 2, of the following special case of a theorem of Sard [2, Theorem 4.1, p. 885] will be used in the proof of Theorem 2.

THEOREM (SARD). Let $M$ be a region in $E^{n}$. If $f: M \rightarrow E^{n}$ is $C^{\prime}$, then the measure of $f\left(Z^{*}\right)$ is zero ( $D$ is a domain and $Z^{*}$ is the set where the Jacobian is zero).

Corollary 2. Let $M$ and $N$ be $C^{\prime} n$-manifolds. If $f$ is $C^{\prime}, f: M \rightarrow N$, then $f\left(Z^{*}\right)$ contains no open sets $\left(Z^{*}\right.$ is the critical set of $f$; i.e., the set where the linear transformation $d f$ is singular).

THeorem 2. Let $M$ and $N$ be $C^{\prime}$ oriented $n$-manifolds and $f: M \rightarrow N$ a $C^{\prime}$ mapping. Assume that $d f$, as a linear transformation, never reverses sense of $M$ and that $d f$ has rank zero at every interior point of $Z^{*}$ (where $Z^{*}$ is the set of points at which df is singular). Then $f$ is in $F$ (thus by Theorem $1, f$ is quasi-open or $M$ is compact and $f(M)$ is a point).

Proof. Condition (i) is clearly satisfied. Corollary 2 shows that (ii) is satisfied since $Z \subset Z^{*}$. Finally, (iii) follows immediately from the rank condition on $d f$.

The following is an example of a $C^{\prime}$ mapping of $E^{n}$ into $E^{n}$ which 
has a non-negative Jacobian and which has rank one on $Z^{*}$ and which is not quasi-open. Thus Theorem 2 is sharp in this sense. Let $R$ be the set of points for which $r^{2}=x_{1}^{2}+\cdots+x_{n}^{2}<4$. Let $u_{k}=\left(r^{2}-1\right)^{2} x_{k}$, $k=1,2, \cdots, n$ when $r \geqq 1$. Let $u_{1}=C\left(1-r^{2}\right), C>18$ and $u_{k}=0$, $k=2,3, \cdots, n$ when $0 \leqq r \leqq 1$. For the $R$ chosen above one has that the image of the origin is not interior to $f(R)$ and thus $f$ is not quasiopen. The verification of the other properties of the example is left to the reader. In fact, the maximum modulus on $\bar{R}$ is achieved at an isolated interior point, the origin. The original topological example for these purposes is due to G. S. Young.

Theorems 1 and 2 either contain or simplify some of the theorems in [3] and are related to some of the material in [6].

\section{BIBLIOGRAPHY}

1. P. Alexandroff and H. Hopf, Topologie I, Berlin, 1938.

2. Arthur Sard, The measure of the critical values of differentiable maps, Proc. Amer. Math. Soc. vol. 48 (1942) pp. 883-890.

3. C. J. Titus and G. S. Young, A Jacobian condition for interiority, Michigan Math. J. vol. 1 (1952) pp. 89-94.

4. G. T. Whyburn, Topological analysis, Princeton University Press, 1958.

5. - Quasi-open mappings, Rev. Math. Pures Appl. vol. 2 (1957) pp. 47-52.

6. G. S. Young, Extension of Liouville's theorem to n-dimensions, Math. Scand. vol. 6 (1958).

UNIVERSITY OF MichigAN 